\section{Nonalcoholic Wernicke Encephalopathy with Extensive Cortical Involvement: Cortical Laminar Necrosis and Hemorrhage Demonstrated with Susceptibility-Weighted MR Phase Images}

Wernicke encephalopathy (WE) is an acute neuropsychiatric condition due to an initially reversible brain lesion caused by depleted intracellular thiamine levels in neurons. ${ }^{1}$ Most of the affected patients are malnourished alcoholics; however, WE has also been associated with other conditions. The typical MR imaging features are symmetric hyperintensity on T2-weighted images in the medial thalami, mamillary bodies, tectal plate, periaqueductal area, and floor of the fourth ventricle. ${ }^{1}$ Atypical MR imaging findings, such as signal-intensity abnormalities in cranial nerve nuclei, cerebellum dentate nuclei, vermis, putamina, red nuclei, caudate nuclei, splenium, and cerebral cortex, have been more frequently described in nonalcoholic WE. ${ }^{1,2}$ The cortical involvement in WE is atypical and relatively rare, being associated with a worse prognosis due to potentially irreversible brain damage. Zuccoli et $\mathrm{al}^{1}$ reported only 1 case with cortical involvement in a series of 56 patients with WE.

We report a 27-year-old woman with Crohn disease who underwent total colectomy due to partial intestinal occlusion and severe disease activity. Two weeks after total parenteral nutrition, she developed aphasia, diplopia, seizures, and acute confusional state. The neurologic examination revealed horizontal nystagmus, bilateral sixth and seventh nerve palsies, tetraparesis, and increased deep tendon reflexes with symmetric spasticity in both upper limbs. Bilateral Babinski reflexes were observed. MR imaging showed symmetric and bilateral hyperintensities on T2-weighted, fluid-attenuated inversion recovery (FLAIR), and diffusion-weighted images involving the hypoglossal, abducens and facial nuclei, medial right thalami, tectum of the midbrain, and extensive symmetric cortical abnormalities in the frontal lobes. The diagnosis of WE was suggested, and treatment with intravenous thiamine was started. Neurologic manifestations such as mental confusion, seizures, cranial nerve palsies, and nystagmus partially improved in the following 2 weeks, except for aphasia and tetraparesis, with spasticity mainly in the upper limbs, confirming the worse prognosis of the cortical lesions. Follow-up brain MR imaging 3 weeks later revealed improvement of most of the lesions. However, the areas of signal-intensity abnormality in the cortex of the frontal lobes persisted, revealing now peripheral high signal intensity on T1weighted images (cortical laminar necrosis) and extensive areas of marked low-signal-intensity susceptibility-weighted MR phase images (SWI) (blood deposits) (Fig 1).

This patient had nonalcoholic WE demonstrating atypical MR imaging findings and rare extensive cortical frontal lesions, progressing to cortical laminar necrosis and hemorrhage. To the best of our knowledge, cortical laminar necrosis and hemorrhage demonstrated with SWI in patients with WE has not been previously reported. SWI

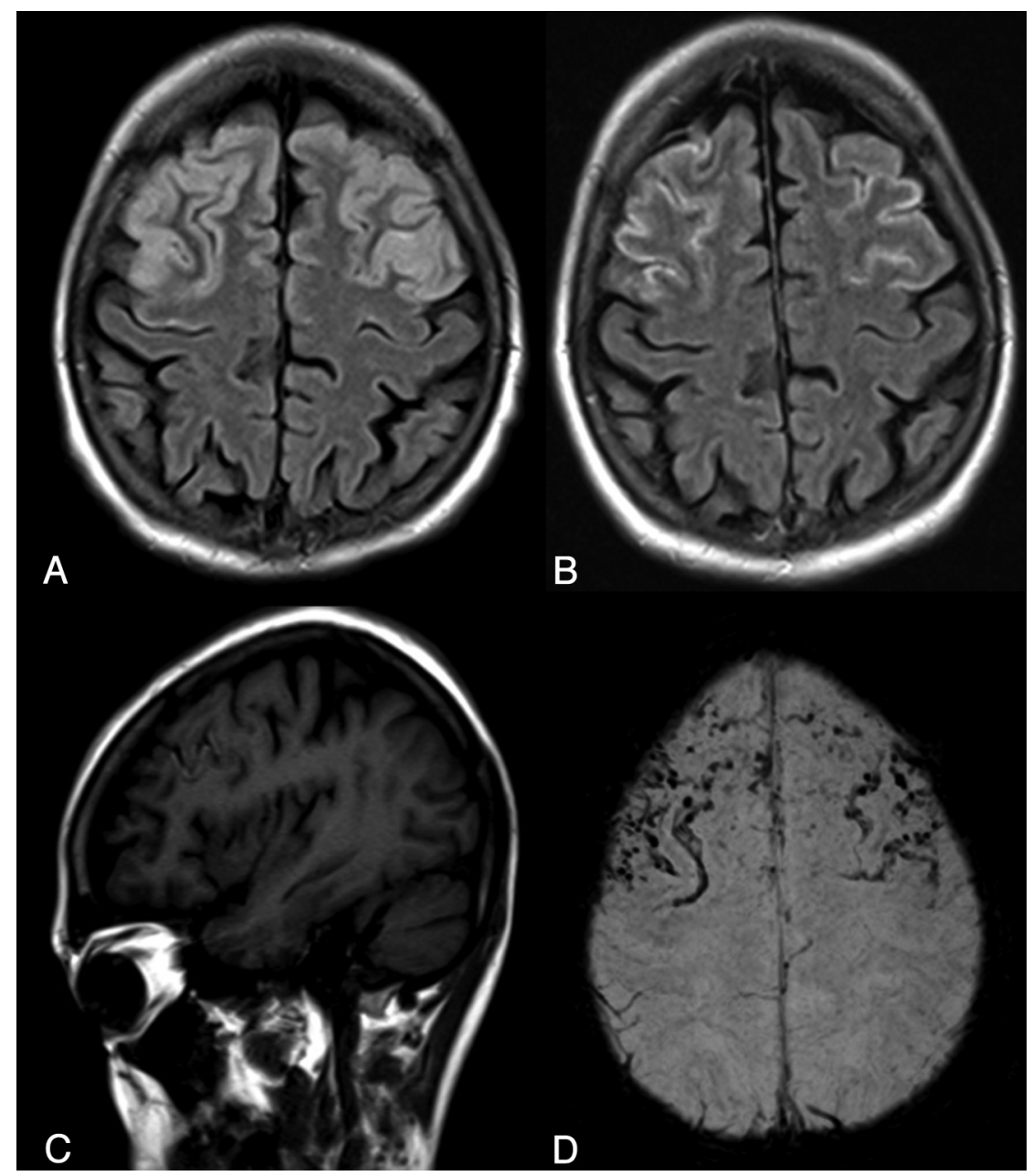

Fig 1. $A$, Axial FLAIR image shows extensive bilateral frontal cortical high signal intensity. B, Follow-up FLAIR image demonstrates significant improvement of the cortical lesions. $C$, Sagittal T1-weighted image shows cortical linear hyperintensities in the right frontal lobe (cortical laminar necrosis). D, SWI minimum-intensity-projection image shows extensive linear and focal areas of marked low signal intensity along the cortical margins in frontal lobes (hemosiderin/ferritin deposits) 
is highly sensitive and superior to $\mathrm{T} 2^{*}$-weighted gradient-echo sequences to even traces of paramagnetic substances, such as blood products and calcifications. ${ }^{3}$ In a previous study of pediatric patients with cortical laminar necrosis evaluated with SWI, only $20 \%$ of the patients had signals of laminar or dotted hemorrhage. ${ }^{4}$ In conclusion, MR imaging follow-up is essential for monitoring patients with WE with cortical abnormalities due to the risk of irreversibility of lesions, which may progress to cortical laminar necrosis and hemorrhage.

\section{References}

1. Zuccoli G, Santa Cruz D, Bertolini M, et al. MR imaging findings in 56 patients with Wernicke encephalopathy: nonalcoholics may differ from alcoholics. AJNR Am J Neuroradiol 2009;30:171-76

2. Hygino da Cruz LC Jr, Domingues RC, Vilanova I, et al. MR imaging findings in
Wernicke encephalopathy: nonalcoholics may be similar to alcoholics. AJNR Am J Neuroradiol 2010;31:E54

3. de Souza JM, Domingues RC, Cruz LC Jr, et al. Susceptibility-weighted imaging for the evaluation of patients with familial cerebral cavernous malformations: a comparison with $\mathrm{T} 2$-weighted fast spin-echo and gradientecho sequences. AJNR Am J Neuroradiol 2008;29:154-58

4. Niwa T, Aida N, Shishikura A, et al. Susceptibility-weighted imaging findings of cortical laminar necrosis in pediatric patients. AJNR Am J Neuroradiol 2008;29:1795-98

D.B. Pereira

M.L. Pereira

E.L. Gasparetto Department of Radiology University Federal of Rio de Janeiro Rio de Janeiro, RJ, Brazil 